$10 / 895905211$
9 SANDIA REPORT

SAND95-8527 • UC-405

Unlimited Release

מ. Printed July 1995

\title{
Simulating Living Organisms with Populations of Point Vortices
}

Robert W. Schmieder

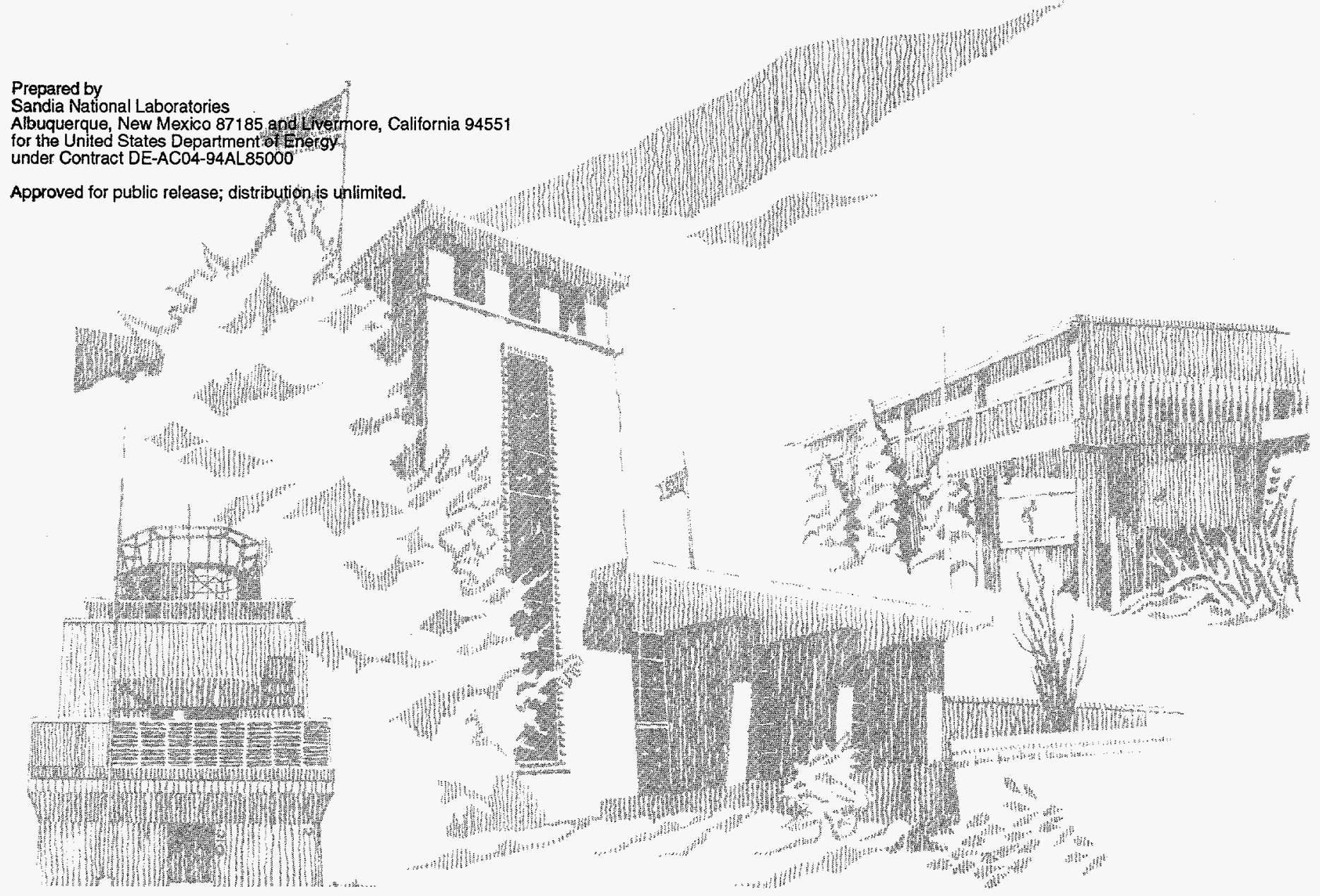


Department of Energy by Sandia Corporation.

NOTICE: This report was prepared as an account of work sponsored by an agency of the United States Government. Neither the United States Government nor any agency thereof, nor any of their employees, nor any of the contractors, subcontractors, or their employees, makes any warranty, express or implied, or assumes any legal liability or responsibility for the accuracy, completeness; or usefulness of any information, apparatus, product, or process disclosed, or represents that its use would not infringe privately owned rights. Reference herein to any specific commercial product, process, or service by trade narne, trademark, manufacturer, or otherwise, does not necessarily constitute or imply its endorsement, recommendation, or favoring by the United States Government, any agency thereof or any of their contractors or subconractors. The views and opinions expressed herein do not necessarily state or reflect those of the United States Government, any agency thereof or any of their contractors or subcontractors.

This report has been reproduced from the best available copy.

\section{Available to DOE and DOE contractors from:}

Office of Scientific and Technical Information

P. O. Box 62

Oak Ridge, TN 37831

Prices available from (615) 576-8401, FTS 626-8401

Available to the public from:

National Technical Information Service

U.S. Department of Commerce

5285 Port Royal Rd.

Springfield, VA 22161 


\section{DISCLAIMER}

Portions of this document may be illegible in electronic image products. Images are produced from the best available original document. 
SAND95-8527

UC-405

Unlimited Release

Printed July 1995

\title{
SIMULATING LIVING ORGANISMS WITH POPULATIONS OF POINT VORTICES
}

\author{
ROBERT W. SCHMIEDER \\ Sandia National Laboratories \\ Livermore, CA 94551 USA \\ rwschmi@ca.sandia.gov
}

\begin{abstract}
We have found that time-averaged images of small populations of point vortices can exhibit motions suggestive of the behavior of individual organisms. As an example, we show that collections of point vortices confined in a box and subjected to heating can generate patterns that are broadly similar to interspecies defense in certain sea anemones. It is speculated that other simple dynamical systems can be found to produce similar complex organism-like behavior.
\end{abstract}




\section{INTRODUCTION}

Complex collective emergent behavior of small populations of simple objects is now a familiar theme in biology. Prime examples are flocking and schooling [Kshatriya and Blake, 1992; Huth and Wissel, 1992], trail formation [Franks, 1989], foraging [Millonas, 1992], synchronized flashing and hibernation, and colonial nest construction. It is usual in such systems to consider time as the independent parameter, and to observe the system configuration evolve in time [Murray, 1993]. Simulation of such emergent behavior is often done using simple physical objects that obey simple behavioral rules [Langton, 1992; Gilpin and Hanski, 1991]. Typically one searches for stable configurations, or configurations that exhibit slow changes: migration, drift, precession, oscillation, and similar patterns [May, 1975; Hoppensteadt, 1992]. Another goal is to attempt the prediction of the emergent behavior from the properties of the individual objects [Gutowitz, 1991]. One encounters in these pursuits the most fundamental question about populations: the origins of order [Kampis, 1991; Kauffman, 1993].

In this paper I point out that under certain conditions, slow configuration changes of small populations of point vortices can mimic the normal behavior of individual living organisms. Point vortices are point-like objects that experience purely transverse mutual forces [Aref, 1979; Aref and Pomphrey, 1982]. They are well-known in theoretical physics, and are useful for simulating fluid dynamics, plasmas, superfluidity and superconductivity, and optics [Pointin and Lundgren, 1976; Kunin, et al., 1992]. In order to generate the behavior we seek, we will place a few ( 5 to 10 ) of these vortices inside a hard-wall box and heat them gently, causing them to move chaotically. The walls of the box provide cooling so the system will eventually attain a quasi-steady state. Then we will make time-integrated images of the population. These images sometimes show quasi-stable structures ("organisms") that move about the box, sometimes interacting with the walls and with each other. By adjusting the numerical parameters, these structures can be made to simulate rather complex behavior of living organisms such as sea anemones. 


\section{SYSTEMS OF POINT VORTICES}

A collection of point vortices is defined by the Hamiltonian

$$
\begin{gathered}
H=\sum_{i \neq j} K_{i j} \log \left(D_{i j}\right) \\
D_{i j}=\sqrt{\left(x_{i}-x_{j}\right)^{2}+\left(y_{i}-y_{j}\right)^{2}}
\end{gathered}
$$

where $\left\{\mathrm{x}_{\mathrm{i}}, \mathrm{y}_{\mathrm{i}}\right\}$ are the canonical coordinates and momenta (which may be considered simply cartesian coordinates of the vortex), and $\mathrm{K}_{\mathrm{ij}}$ are arbitrary. From $\mathrm{H}$ in we find the equations of motion of each vortex:

$$
\begin{aligned}
\frac{d x_{i}}{d t} & =\sum_{j \neq i}^{N} K_{i j} \frac{\left(y_{i}-y_{j}\right)}{D_{i j}} \\
\frac{d y_{i}}{d t} & =-\sum_{j \neq i}^{N} K_{i j} \frac{\left(x_{i}-x_{j}\right)}{D_{i j}}
\end{aligned}
$$

This system produces motions in which the vortices revolve forever around themselves, a kind of complex multiple egg-beater pattern. Because $\mathrm{H}$ is constant, the patterns remain confined within a finite region determined by the total energy. If, however, we add energy to the vortices, their trajectories expand; the circulating motions have larger and larger radii, and this can continue without limit; the vortices spiral forever outward.

Vortex heating can be simulated numerically by taking finite step sizes in the integration of the equations of motion. The reason for this is that an exact solution would produce the exactly adiabatic circular motion, while finitedifference solutions will produce linear path increments extending outside the circular orbits, corresponding to higher energy. Physically, this process is equivalent to a centrifugal force that heats the vortices. In numerically solving the equations of motion we simply can't avoid heating of the vortices, and they automatically spiral outward.

We can, however, confine the vortices by introducing a hard wall around them. When a vortex hits the wall, it is given a small inward kick, thereby reducing its energy and keeping it confined within the box. The wall is therefore 
"cooling" the vortices, exactly balancing any amount of heating.

Vortex cooling can be simulated by simply repositioning an impinging vortex slightly away from the wall, to the inside. Physically this is equivalent to giving the vortex an impulse when it strikes the wall. Typically what happens is that the vortex then slides to the side and strikes the wall again, and is again given an impulse. Eventually the vortex may drift away from the wall.

From these considerations we adopt the following finite difference equations to represent the heated vortices confined in a rectangular box:

$$
\begin{aligned}
& x_{i}(t+1)=x_{i}(t)+\delta s \sum_{j \neq i}^{N} K_{i j} \frac{\left(y_{i}-y_{j}\right)}{D_{i j}}-\delta z h\left(x_{i}-x_{w}\right) \\
& y_{i}(t+1)=y_{i}(t)-\delta s \sum_{j \neq i}^{N} K_{i j} \frac{\left(x_{i}-x_{j}\right)}{D_{i j}}-\delta z h\left(y_{i}-y_{w}\right)
\end{aligned}
$$

where $\mathrm{x}_{\mathrm{w}}$ and $\mathrm{y}_{\mathrm{w}}$ represent the walls and $\delta \mathrm{s}$ and $\delta \mathrm{z}$ are arbitrary (but small). The heaviside function $h(x)=0,1 / 2,1 \quad(x<,=,>0)$ ensures that so long as the vortex is inside the box, no cooling occurs. The time $t$ is assumed to be integral.

In order to reduce the number of free parameters, we arbitrarily adopt the following constraint: The $\mathrm{N}$ vortices will be identified as either male (M) or female (F). A population of $n M$ and $m F$ vortices will be written $P=\{n, m\}$. We assume that only 4 kinds of interactions take place: $(M, M),(M, F),(F, M)$, and (F,F). The NxN matrix $\mathrm{K}_{\mathrm{ij}}$ is therefore taken to have only 4 independent values:

$$
\mathrm{K}_{\mathrm{ij}}=\mathrm{S}_{1} \delta_{\mathrm{iM}} \delta_{\mathrm{jM}}+\mathrm{S}_{2} \delta_{\mathrm{iF}} \delta_{\mathrm{jM}}+\mathrm{S}_{3} \delta_{\mathrm{iM}} \delta_{\mathrm{jF}}+\mathrm{S}_{4} \delta_{\mathrm{iF}} \delta_{\mathrm{jF}}
$$

For instance, a population of $\mathrm{P}=\{2,3\}$ vortices will have $\mathrm{K}_{\mathrm{ij}}$ given by

\begin{tabular}{l|lllll} 
ilj & $M$ & $M$ & $F$ & $F$ & $F$ \\
\hline$M$ & $\ldots$ & $S_{1}$ & $S_{2}$ & $S_{2}$ & $S_{2}$ \\
$M$ & $S_{1}$ & $\ldots$ & $S_{2}$ & $S_{2}$ & $S_{2}$ \\
$F$ & $S_{3}$ & $S_{3}$ & $\ldots$ & $S_{4}$ & $S_{4}$ \\
$F$ & $S_{3}$ & $S_{3}$ & $S_{4}$ & $\cdots$ & $S_{4}$ \\
$F$ & $S_{3}$ & $S_{3}$ & $S_{4}$ & $S_{4}$ & $\cdots$
\end{tabular}

The vectors $\mathbf{P}=\{\mathrm{n}, \mathrm{m}\}$ and $\mathbf{S}=\left\{\mathrm{S}_{1}, \mathrm{~S}_{2}, \mathrm{~S}_{3}, \mathrm{~S}_{4}\right\}$ are sufficient to define the system. We emphasize that this is an arbitrary special case; $\mathrm{K}_{\mathrm{ij}}$ actually allows $\mathrm{N}^{2}-\mathrm{N}$ different values. $\mathrm{K}_{\mathrm{ii}}$ is not relevant, since vortices have no self-interaction. 


\section{SIMULATING ORGANISM BEHAVIOR}

Populations of vortices as described above exhibit very complex, even chaotic, motions. However, we have found that under certain conditions we can produce motions that are quasi-periodic. We take advantage of this circumstance to make the transition from population behavior to organism behavior. This is done by taking a "time-exposure" image of the population.

The position of each vortex in the box is represented as a dot. Motion of the vortex is simulated by putting a dot at the new position and erasing the old dot. If, however, we display dots at, say, the previous 100 positions, we see wormlike patterns. To the extent that the step size is small, these patterns appear relatively smooth, and they appear to move relatively smoothly. If the motion is smooth but chaotic, the patterns appear as wandering wormlike curves. If, however, the motion is quasi-periodic, the worms "chase their tails," and the patterns appear on the screen as ringlike figures that move relatively slowly, or not at all. Sometimes the rings move freely within the box, and sometimes they are stabilized on the wall, appearing much like a "flat tire."

The representation of vortex trajectories as time-integrated images converts the set of rapidly moving points into slowly moving geometric figures. These metastable patterns exhibit behavior totally unlike the behaviors of the point vortices themselves. The patterns appear on the screen as "organisms" that move, interact, evolve, appear, and dissappear. Sometimes one pattern will "ingest" another, sometimes "destroy" another. While the underlying reality is the motion of the point vortices, it is the metastable patterns that we find interesting. We have found a few examples of such patterns that are suggestive of actual biological organisms, which we now describe.

\section{SIMULATED ORGANISMS}

Figure 1 shows two frames of a population of $2 \mathrm{M}$ and $3 \mathrm{~F}$ vortices, i.e., $\mathbf{P}=\{2,3\}$. The interactions are set to $\mathbf{S}=\{1,-.7, .7,-1\}$. Thus, $\{\mathrm{M}, \mathrm{M}\}$ and $\{\mathrm{F}, \mathrm{M}\}$ pairs "rotate" around each other, while $\{\mathrm{M}, \mathrm{F}\}$ and $\{\mathrm{F}, \mathrm{F}\}$ pairs "antirotate." The 5 vortices were initially randomly positioned in the square box, and then allowed to move according to the finite-difference equations given above. After a very long time, the vortices separated into two "organisms," one (left) with $1 \mathrm{M}$ and 1 F, i.e., $\mathbf{p}=\{1,1\}$, the other with $1 \mathrm{M}$ and $2 \mathrm{~F}$, i.e., $\mathbf{p}=\{1,2\}$. These organisms were 
stabilized against the wall, sliding unpredictably to the left or right. Eventually the right organism was stabilized in the corner, from which it did not escape. For a very long time the left organism did an irregular, rather tentative back-andforth dance on the wall, staying beyond a critical distance. Suddenly, without any warning, it moved closer, and extended a long filament toward the organism in the corner.

The behavior shown in Fig. 1, while not accurate in all details, is broadly reminiscent of the behavior of certain living organisms. For instance, it is known that certain cnidarians such as Corynactis californica have very agressive defense mechanisms [Morris, et al., 1980, Chao, 1975]. When approached by another anemone (Anthopleura elegantissima), Corynactis will extend a mesentary filament that stings the victim, causing its death and complete disintegration of the organism.

By adjusting the interaction parameters $\mathbf{S}$, a variety of interesting behaviors can be produced. Figure 2 shows the same population $\mathbf{P}=\{2,3\}$ using $\mathbf{S}=\{1,-.7, .7,-$ 1) and $S=\{1,-.1,1,-1\}$. In Fig. 2(a), the interaction results in the total disintegration of both organisms. In Fig. 2(b), the organisms merge to form a single combined organism. This is vaguely reminescent of fertilization, or ingestion by protozoa. Note that because of the wall interaction, the equations are not symmetric with respect to time-reversal; we cannot run the calculation backward to simulate fission.

We have also found patterns that spontaneously go into stable oscillation. Figure 3 shows one such system, consisting of $\mathbf{P}=\{2,1\}, \mathbf{S}=\{1.4,-1.3,1.4,-1.3\}$. The two sequences (a) and (b) are two independent frame sequences generated with exactly the same population, starting from two different (random) initial configurations. After a long transient, each configuration settled to the quasiperiodic patterns shown in Fig. 3(a),(b). In both cases, one organism is stabilized in the corner, and is repeatedly attacked by the other. The attacker waits a long time at a safe distance, then suddenly attacks. It is completely destroyed by the defender, but manages to reassemble at the safe distance, where it effects repairs and prepares for the next attack. This periodic behavior appeared to persist forever. The period was relatively well-defined.

As a final example, Figure 4 shows a classic pursuit and flight relationship. The linear wormlike organism crawls along the wall toward the large, circular ringlike organism in the corner. When the worm reaches the ring, the latter is temporarily disrupted and flees along the other wall into the opposite corner. 
The worm, however, pursues it, and the sequence repeats. The net result is that the pair of organisms progress in this start-stop motion counterclockwise forever around the inside of the box. The most immediate image evoked by this simulation is of a cat chasing a grasshopper, or perhaps of a grasshopper pestering a sleepy cat.

\section{DISCUSSION}

We emphasize that these phenomena appear only when the vortex populations are confined in the box and heated (and cooled at the walls). We expect any collection of objects to expand when heated. Confining the collection while maintaining heating is equivalent to cooling the objects at the walls. Thus, we obtain a dynamic equilibrium between heating and cooling. In general we expect to find equilibia in such systems that are very different from the equilibria obtained in adiabatic systems. A very general response of systems under these conditions is ordering: the system undergoes phase transitions that increase internal order. Examples are domain formation, crystallization, and coherent motion. Apparently what we see in the populations of point vortices is just this: formation of "organisms" with their own simplified motions.

We also point out the relevance of connectivity to these simulations. There is a growing understanding that connectivity in ecosystems is a crucial determinant of complexity [Farmer, 1990; Green, 1993, 1994]. If the ecosystem is weakly connected or if it is strongly connected, it is predictable, but near a critical value of connectivity, it is inherently chaotic and unpredictable. The relevance to the present work is through the couplings $\mathrm{K}_{\mathrm{ij}}$, which are a measure of the connectivity. The stonger interaction in Fig. 2(a) resulted in chaotic disruption of the organisms, while the weaker interaction in Fig. 2(b) resulted in their nearly unperturbed fusion. Thus, we should not be surprised that the behavior of the simulated organisms should depend sensitively on the couplings $\mathrm{K}_{\mathrm{ij}}$. In fact, we have found cases in which the alteration of $S_{2}$ by as little as 0.1 percent completely alters the gross emergent behavior of the population.

These simulations are not sufficiently accurate to suggest they would be useful in understanding behavior, and we see no causative relationship between these simulations and real organisms. However, they are sufficiently remarkable that they probably are more than simply amusing. With the flexibility provided by the interactions $S_{i j}$, we would expect a wide variety of behaviors suggestive of living organisms. Since these behaviors emerge from the simple behavior of 
more fundamental objects (the point vortices), they are manifestly linked to a common origin. Exploration of this kind of system might bring insight into the range of phenomena one might expect from populations of simple physical objects.

Based on the results of this paper, we therefore expect to find a wide range of new and interesting epi-phenomena associated with small populations of confined objects subject to heating. Point vortices provide one interesting system with which to study these phenomena. We might expect similar phenomena from populations of oscillators, pendulums, dipoles, switches, hinges, chains, and similar objects.

\section{REFERENCES}

Aref, H. 1979. Motion of three vortices. Phys. Fluids 22:393-400.

Aref, H. and N. Pomphrey. 1982. Integrable and chaotic motions of four vortices.

I. The case of identical vortices. Proc. Roy. Soc. Lond. A380:59-387.

Brillouin, L. 1962. Science and Information Theory. 2nd Ed. Academic Press, New York.

Burns, T. P. 1994. On the fittedness of organisms and the ascendency of ecosystems: Toward a hierarchical model of network development. J. Theor. Biol. 170:115-127.

Chao, C. 1975. Inter-specific agression between three sympatric anemones, Anthopleura elegantissima, Metridium senile, and Corynactis california. Res. Paper Biol 175h, Hopkins Marine Sta., Stanford Univ., Pacific Grove, CA.

DeAngelis, D. L., ed. 1992. Individual-based Approaches in Ecology. Chapman and Hall, London.

Farmer, J. D. 1990. A Rosetta Stone for Connectionism. In: S. Forrest, ed. Emergent Computation: Self-Organizing, Collective, and Cooperative Phenomena in Natural and Artificial Computing Networks. reprinted from Physica D 42 as special issue, pp. 153-187.

Franks, N. R. 1989. Army Ants: A Collective Intelligence. American Scientist 77:139-145.

Gilpin, M. and I. Hanski. 1991. Metapopulation Dynamics: Empirical and Theoretical Investigations. Academic Press, London.

Green, D. G. 1993. Emergent behavior in biological systems. In: Complex Systems

- From Biology to Computation, D. G. Green and T. J. Bossomaier, eds., IOS 
Press, Amsterdam, pp. 24-35.

Green, D. G. 1994. Connectivity and complexity in landscapes and ecosystems.

Pac. Conservation Biology, in press.

Gutowitz, H. 1991. Cellular Automata. Reprinted from Physica D 45 as special issue.

Hoppensteadt, F. C. 1982. Mathematical Methods of Population Biology.

Cambridge University Press, Cambridge.

Huth, A. and C. Wissel. 1992. The Simulation of the Movement of Fish Schools.

J. Theor. Biol. 156:365-385.

Kampis, G. 1991. Self-Modifying Systems in Biology and Cognitive Science.

Pergamon Press, Oxford.

Kauffman, S. A. 1993. The Origins of Order. Self-Organization and Selection in Evolution. Oxford University Press, New York.

Kshatriya, M. and R. W. Blake. 1992. Theoretical Model of the Optimum Flock Size of Birds Flying in Formation. J. Theor. Biol. 157:135-174.

Kunin, I. A., F. Hussain, X. Zhou, and S. J. Prishepione. 1992 Centroidal frames in dynamical systems. I. Point vortices. Proc. R. Soc. Lond. A439:441-463.

Langton. C., ed. 1992. Artificial Life II. Addison-Wesley Publ. Co., Redwood City, CA. Also: 1989. Artificial Life (I).

Lomnicki, A. 1988. Population Ecology of Individuals. Princeton University Press, Princeton, NJ.

May, R. M. 1975. Stability and Complexity in Model Ecosystems, 2nd Ed., Princeton University Press, Princeton.

Millonas, M. M. 1992. A Connectionist Type Model of Self-Organized Foraging and Emergent Behavior in Ant Swarms. J. Theor. Biol. 159:529-552.

Morris, R. H., D. P. Abbott, and E. C. Haderlie. 1980. Intertidal Invertebrates of California. Stanford Univ. Press.

Murray, J. D. 1993. Mathematical Biology, Springer-Verlag, Berlin.

Pointin, Y. B. and T. S. Lundgren. 1976. Statistical mechanics of two-dimensional vortices in a bounded container. Phys. Fluids 19:1459-1470. 


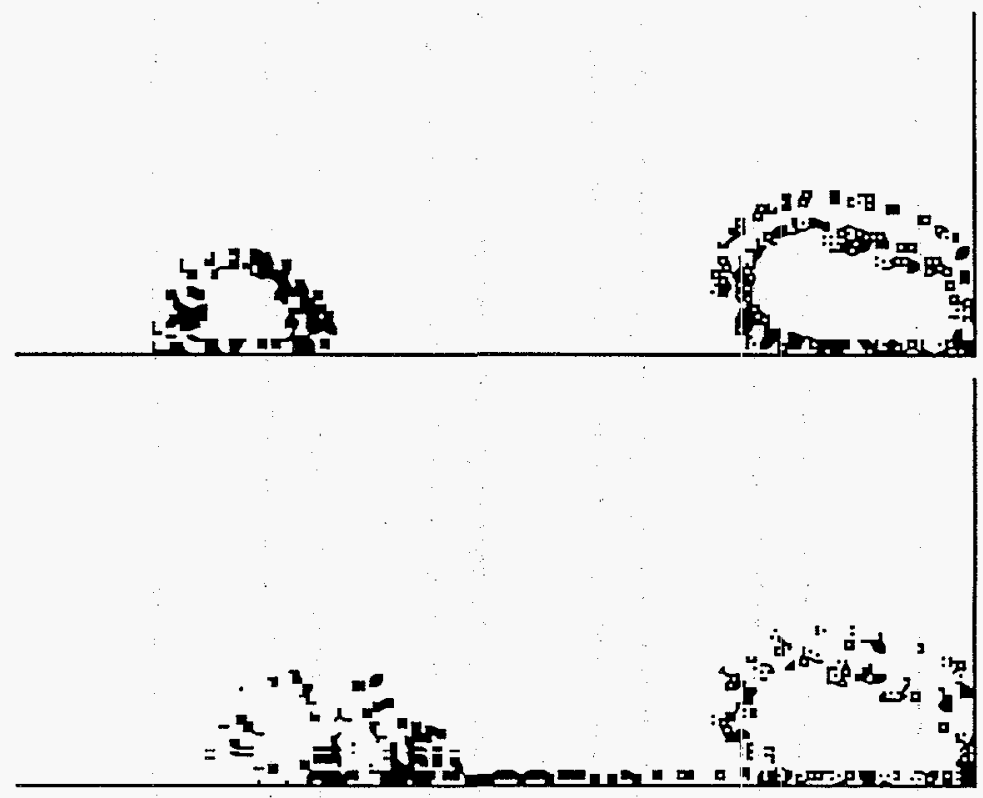

Figure 1 - Simulated filament extension. The organism on the right is confined to the corner, while the one on the left does a slow, irregular dance on the wall in front of it. Without warning, the left organism extends a long filament toward the right one, suffering some disruption in the process. 

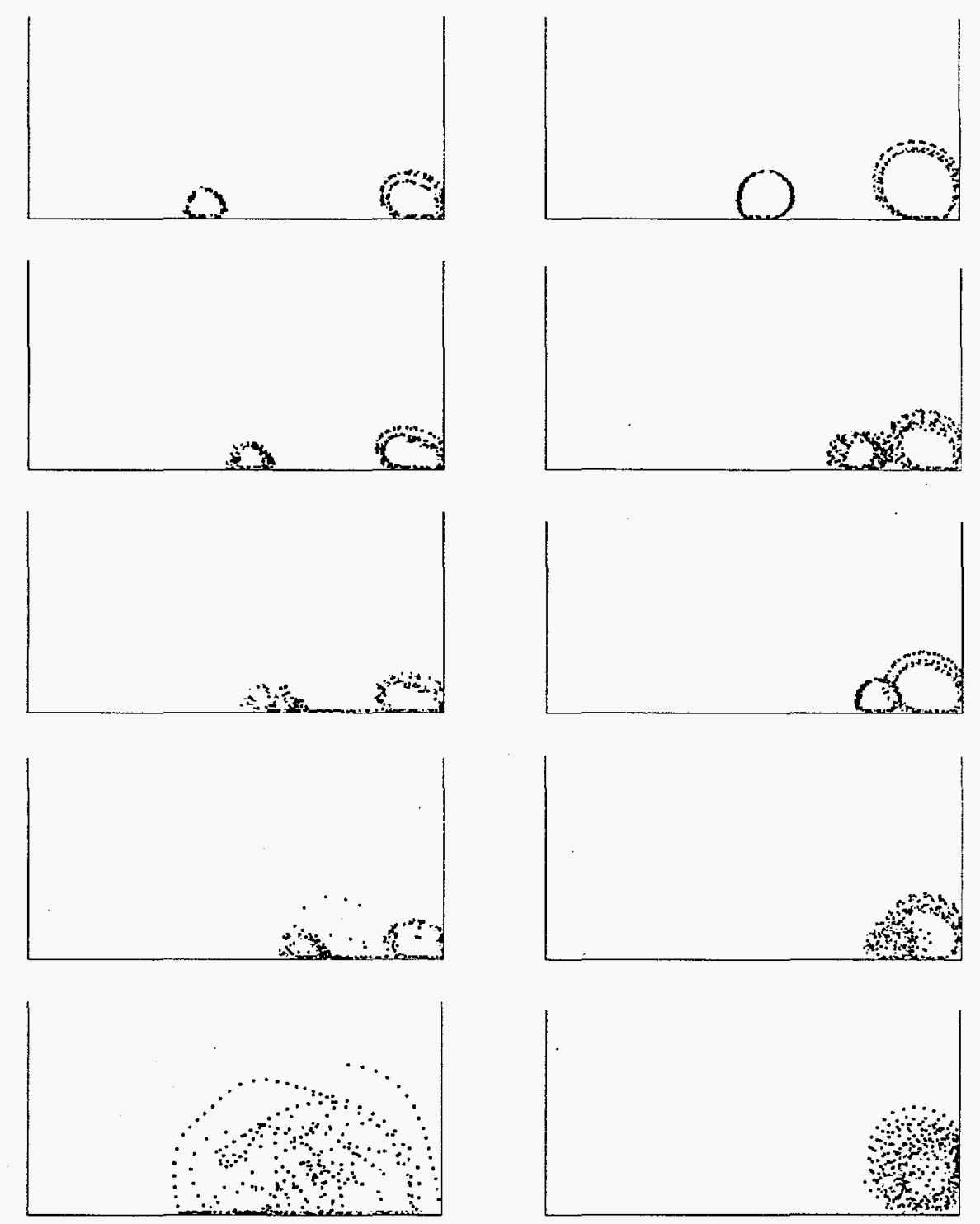

(a)

(b)

Figure 2 - (a) Simulated filament-induced disintegration. $\mathbf{P}=\{2,3\}$, $\mathbf{S}=\{1,-.7, .7,-1\}$; (b) Simulated fusion. $\mathbf{P}=\{2,3\}, \mathbf{S}=\{1,-.1, .1,-1\}$. 


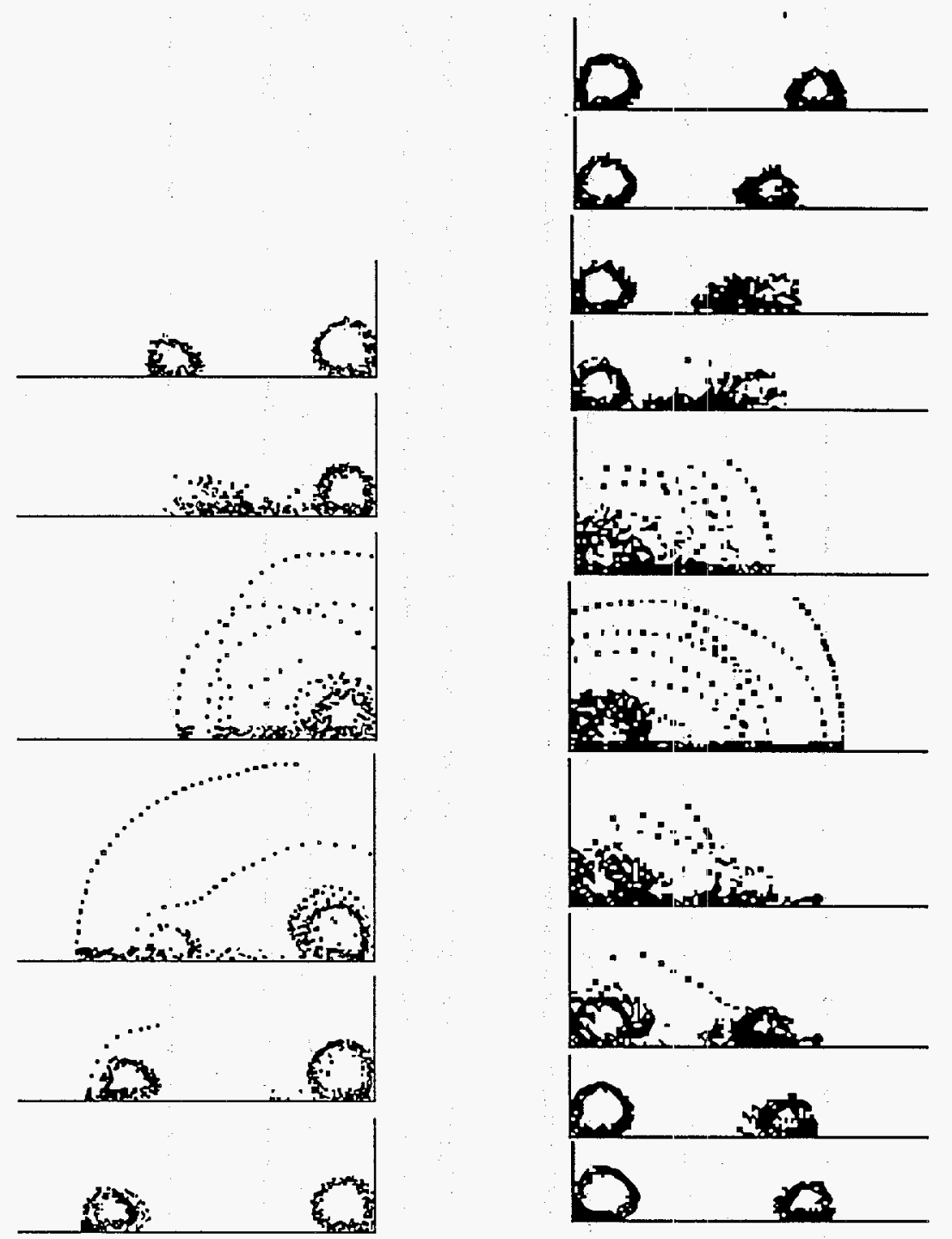

(a)

(b)

Figure 3 - Simulated periodic attack and defense. The two sequences (a) and (b) were generated independently with exactly the same population, starting from different random initial configurations. $\mathbf{P}=\{2,2\}, \mathbf{S}=\{1.4,-1.3,1.4,-1.3\}$. 


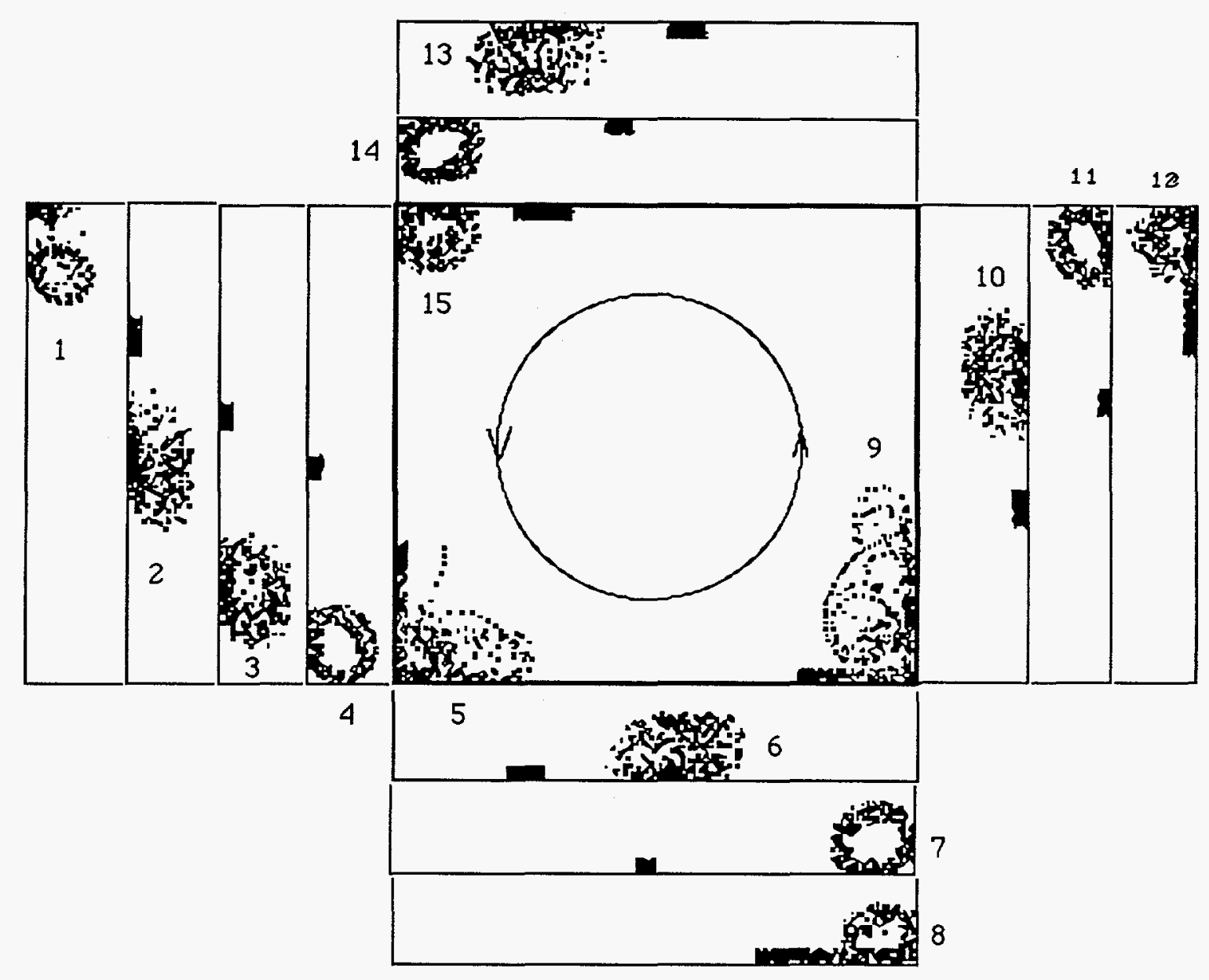

Figure 4 - Simulated pursuit and flight. The small straight organism chases the circular organism forever counterclockwise around the box. Extra frames around the box show the configuration at various times. The numbers indicate the frame sequence. $P=\{2,1\}, S=\{1, .2,-1,-1\}$. 


\section{UNLIMITED RELEASE}

INITIAL DISTRIBUTION:

\begin{tabular}{|c|c|}
\hline MS0724 & D. L. Hartley, 6000 \\
\hline MS9001 & J. C. Crawford, 8000 \\
\hline MS9002 & P. N. Smith, 8500 \\
\hline MS9003 & D. L. Crawford, 8900 \\
\hline MS9004 & M. E. John, 8100 \\
\hline MS9005 & J. B. Wright, 5300 \\
\hline MS9006 & G. E. Ives, 5300 \\
\hline MS9037 & R. J. Detry, 8200 \\
\hline MS9054 & W. J. McLean, 8300 \\
\hline MS9103 & G. Thomas, 8111 \\
\hline MS9105 & L. A. Hiles, 8400 \\
\hline MS9108 & T. M. Dyer, 8800 \\
\hline MS9141 & S. Johnston, 8103 \\
\hline MS9163 & W. Bauer, 8302 \\
\hline MS9201 & L. D. Brandt, 8112 \\
\hline MS9201 & R. J. Gallagher, 8114 \\
\hline MS9214 & L. M. Napolitano, Jr., 8117 \\
\hline MS9214 & C. L. Bisson, 8117 \\
\hline MS9214 & M. E. Colvin, 8117 \\
\hline MS9214 & E. Friedman-Hill, 8117 \\
\hline MS9214 & R. S. Judson, 8117 \\
\hline MS9214 & W. P. Kegelmeyer, 8117 \\
\hline MS9214 & C. F. Melius, 8117 \\
\hline MS9214 & J. C. Meza, 8117 \\
\hline MS9214 & R. W. Schmieder, 8117 (10) \\
\hline MS9401 & R. C. Wayne, 8700 \\
\hline MS9403 & M. I. Baskes, 8712 \\
\hline MS9410 & J. C. Swearengen, 8113 \\
\hline MS9901 & L. A. West, 8600 \\
\hline MS9904 & W. D. Wilson, 8703 \\
\hline
\end{tabular}

MS9021 Technical Communications Department, 8535 for OSTI (10)

MS9021 Technical Communications Department, 8535/Technical Library, MS0899, 13414

MS0899 Technical Library, 13414 (4)

MS9018 Central Technical Files, 8523-2 (3) 